\title{
SYMMETRIES OF AN EXTENDED HUBBARD MODEL
}

\author{
BIANCA LETIZIA CERCHIAI \\ Sektion Physik, Universität München, LS Prof. Wess \\ Theresienstr. 37, D-80333 München, Germany \\ E-mail: Bianca.Cerchiai@physik.uni-muenchen.de \\ PETER SCHUPP \\ Sektion Physik, Universität München, LS Prof. Wess \\ Theresienstr. 37, D-80333 München, Germany \\ E-mail: Peter.Schupp@physik.uni-muenchen.de
}

\begin{abstract}
The Hamiltonian for an extended Hubbard model with phonons as introduced by A. Montorsi and M. Rasetti is considered on a $D$-dimensional lattice. The symmetries of the model are studied in various cases. It is shown that for a certain choice of the parameters a superconducting $S U_{q}(2)$ holds as a true quantum symmetry, but only for $D=1$.
\end{abstract}

1. Introduction. In this article we are going to study extensions of the Hubbard model on a $D$-dimensional lattice and their symmetries.

The Hubbard model was originally introduced in [1] and is the simplest one describing systems of itinerant interacting electrons in solid-state physics. Its importance is mainly due to the fact that it is supposed to describe high-temperature superconductivity.

Since the work of Yang and Zhang [2,3] it has been known that the Hubbard model has a $(S U(2) \times S U(2)) / \mathbf{Z}_{2}$-symmetry. This symmetry is the product of two separate $S U(2)$ symmetries: a "magnetic" symmetry, which accounts for the (antiferro-)magnetic properties of the electron system, and a "superconductive" symmetry, which is supposed to give rise to superconductivity when it is broken.

Montorsi and Rasetti investigated whether the symmetry of the standard Hubbard model can be generalized to a quantum group symmetry of an extended Hubbard model. In [4] they claim the existence of a "superconductive" $S U_{q}(2)$-quantum group symmetry and an unchanged "magnetic" $S U(2)$-symmetry for a Hubbard model with non-local phonon interaction. We are able to verify this symmetry for a particular extension of the Hubbard model, but find it to be restricted to 1-dimensional lattices. Moreover,

1991 Mathematics Subject Classification: Primary 81R50; Secondary 82D55, 82B20.

The paper is in final form and no version of it will be published elsewhere. 
the Quantum Symmetric Hubbard Model is not the standard Hubbard Model with Phonons.

2. The extended Hubbard model. The Hamiltonian of the extended Hubbard model is given by $[4,5]$ :

$$
H_{H u b}=H_{e l}^{(l o c)}+H_{p h}+H_{e l-p h}^{(l o c)}+H_{e l-p h}^{(n o n-l o c)}
$$

where

$$
\begin{aligned}
& H_{e l}^{(l o c)}=u \sum_{i} n_{i \uparrow} n_{i \downarrow}-\mu \sum_{i, \sigma} n_{i \sigma} \\
& H_{p h}=\sum_{i}\left(\frac{\vec{p}_{i}^{2}}{2 M}+\frac{1}{2} M \omega^{2} \vec{x}_{i}^{2}\right) \\
& H_{e l-p h}^{(l o c)}=-\vec{\lambda} \cdot \sum_{i}\left(n_{i \uparrow}+n_{i \downarrow}\right) \vec{x}_{i} \\
& H_{e l-p h}^{(n o n-l o c)}=\sum_{\langle i<j\rangle} \sum_{\sigma} T_{i j} a_{j \sigma}^{\dagger} a_{i \sigma}+h . c .
\end{aligned}
$$

with $T_{i j}$ given by

$$
T_{i j}=t e^{-\hbar \zeta \vec{R}_{i j} \cdot \vec{\kappa}} \exp \left\{\zeta \vec{R}_{i j} \cdot\left(\vec{x}_{i}-\vec{x}_{j}\right)+i \vec{\kappa} \cdot\left(\vec{p}_{i}-\vec{p}_{j}\right)\right\}
$$

In these expressions the Hamiltonian depends on the operators $a_{i \sigma}, a_{i \sigma}^{\dagger}, \vec{p}_{i}, \vec{x}_{i}$, where $a_{i \sigma}, a_{i \sigma}^{\dagger}$ are fermionic annihilation/creation operators for an electron with spin $\sigma=\{\uparrow, \downarrow\}$ at site $i$, while $\vec{p}_{i}, \vec{x}_{i}$ are momentum and displacement operators for an ion at site $i$ with the usual commutation relations.

The Hamiltonian also depends on $\vec{R}_{i}$ which gives the rest position of the the ion at site $i$. Note that the expression $\vec{R}_{i j} \equiv \frac{\left(\vec{R}_{j}-\vec{R}_{i}\right)}{\left|\vec{R}_{i}-\vec{R}_{j}\right|}$ always has the same module and that in the one-dimensional case it just amounts to a sign. $\left|\vec{R}_{i}-\vec{R}_{j}\right|$ is the interatomic distance at equilibrium so that it does not depend on $i, j$. The explanation of the various terms composing $H_{H u b}$ is the following.

$H_{e l}^{(l o c)}$ is the local electron-electron interaction and is the sum of two terms. The first one, the on-site repulsion $u \sum_{i} n_{i \uparrow} n_{i \downarrow}$, is a reminiscent of the Coulomb repulsion between the electrons and is determined by the parameter $u$. The second one, the chemical potential $-\mu \sum_{i, \sigma} n_{i \sigma}$, shows that the Hamiltonian is written in a grand-canonical formalism, and hence the parameter $\mu$ fixes the number of electrons in the lattice.

$H_{p h}$ is the kinetic term for the phonons, which are supposed to be described by a set of decoupled Einstein oscillators with the same frequency $\omega$ and the same mass $M$.

$H_{e l-p h}^{(l o c)}$ is the local phonon-electron interaction term [6,7], which at each site is an attractive force proportional to the number of electrons and to the ion displacement with coupling constant $\vec{\lambda}$.

The crucial term is the non-local one. It is a hopping amplitude, and gives the probability that an electron can jump from one site to another one. Notice that we have retained only the nearest neighbour terms $\langle i j\rangle$ and hence assumed negligible overlap between all other atomic orbitals. In the extended model this amplitude depends from the 
ion displacement and momentum, so that a non-local interaction between the phonons and the ions is present.

Let's study different special cases of the extended Hubbard model and see which models one can recover.

2.1. Overview of the different models contained in the extended Hubbard model. In the following table we give a list of the different models contained in the extended Hubbard model. A more detailed explanation follows in the remarks.

I) Hubbard model with: $\zeta=0$

a) generic case: $\vec{\lambda} \neq 0, \vec{\kappa} \neq 0$

b) with local phonon interaction: $\vec{\lambda} \neq 0, \vec{\kappa}=0 \stackrel{\text { Lang-Firsov-transform. }}{\Longleftrightarrow} \mathrm{c})$

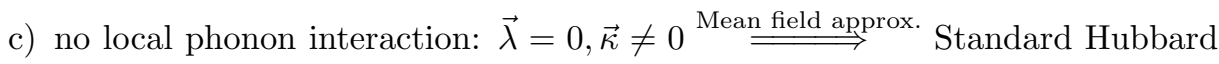
model

d) Standard Hubbard model plus decoupled Einstein oscillators: $\vec{\lambda}=0, \vec{\kappa}=0$

II) Hubbard model with: $\zeta \neq 0$, but $\vec{\kappa}=0$

a) with local phonon interaction: $\vec{\lambda} \neq 0$

b) no local phonon interaction: $\vec{\lambda}=0$

III) General case: $\zeta \neq 0, \vec{\kappa} \neq 0, \vec{\lambda} \neq 0$

Remarks. ad I) When $\vec{\kappa}=0, \zeta=0$, but $\vec{\lambda} \neq 0$ (case Ib) the Hamiltonian $H_{H u b}$ can be used to describe bipolarons [8], a model in which there is only the local electronphonon interaction. Notice that equivalently, it is possible to describe the bipolarons with an Hamiltonian of the type $H_{H u b}$ with $\vec{\lambda}=0$, but $\vec{\kappa} \neq 0$ (case Ic). This can be done by performing a unitary transformation, the Lang-Firsov transformation [9], on $a_{i \sigma}, a_{i \sigma}^{\dagger}, p_{i}, x_{i}$ with a unitary operator

$$
U(\vec{\kappa}) \equiv \exp \left(i \vec{\kappa} \cdot \sum_{l, \sigma} \vec{p}_{l} n_{l \sigma}\right) .
$$

Performing a mean-field approximation on the phonon variables (when $\vec{\lambda}=0$ ), one recovers the standard Hubbard model.

ad II) When $\vec{\kappa}=0$ the Hamiltonian $H_{H u b}$ describes the Hubbard model with phonons added. To see this, remember that the hopping amplitude in (5) is originally defined by Hubbard [1]

$$
T_{i j}=\int d^{D} r \Psi^{*}\left(\vec{r}-\vec{R}_{i}-\vec{x}_{i}\right)\left(-\frac{\hbar^{2} \nabla^{2}}{2 m}\right) \Psi\left(\vec{r}-\vec{R}_{j}-\vec{x}_{j}\right) .
$$

where $\Psi\left(\vec{r}-\vec{R}_{i}\right)$ is the Wannier electron wave function. $T_{i j}$ is a function only of $\vec{a}_{i j} \equiv$ $\left(\vec{R}_{i}+\vec{x}_{i}\right)-\left(\vec{R}_{j}+\vec{x}_{j}\right)$. We approximate the Wannier electron functions with atomic orbitals, which show an asymptotic exponential decay $\Psi(\vec{r}) \sim e^{-\zeta|\vec{r}|}$ and find

$$
\nabla_{\vec{a}_{i j}} T\left(\vec{a}_{i j}\right)=\int d^{D} r \zeta \frac{\left(\vec{r}-\vec{a}_{i j}\right)}{\left|\vec{r}-\vec{a}_{i j}\right|} \Psi^{*}\left(\vec{r}-\vec{a}_{i j}\right) \frac{\hbar^{2} \nabla^{2}}{2 m} \Psi(\vec{r})
$$


Because of the rapid exponential decay of $\Psi(\vec{r})$, we can also neglect $\vec{r}$ in $\left|\vec{r}-\vec{a}_{i j}\right|$ so that [10]

$$
\nabla_{\vec{a}_{i j}} T\left(\vec{a}_{i j}\right)=-\zeta \frac{\vec{a}_{i j}}{\left|\vec{a}_{i j}\right|} T\left(\vec{a}_{i j}\right)
$$

which integrates to $T\left(\vec{a}_{i j}\right)=T_{0} e^{-\zeta\left|\vec{a}_{i j}\right|}$.

$\left|\vec{a}_{i j}\right|=\left|\vec{R}_{i}-\vec{R}_{j}+\vec{x}_{i}-\vec{x}_{j}\right|$ can be approximated by $\left|\vec{x}_{i}-\vec{x}_{j}\right| \ll\left|\vec{R}_{i}-\vec{R}_{j}\right|$ such that [5]

$$
T_{i j}=t \exp \left(-\zeta \frac{\left(\vec{R}_{i}-\vec{R}_{j}\right)}{\left|\vec{R}_{i}-\vec{R}_{j}\right|}\left(\vec{x}_{i}-\vec{x}_{j}\right)\right)
$$

with a new constant $t=T_{0} \exp \left(-\zeta\left|\vec{R}_{i}-\vec{R}_{j}\right|\right)$.

3. Symmetries of the extended Hubbard model. None of the terms added to Hubbard model affects the magnetic $S U(2)$-symmetry. Hence, we shall only be concerned with the superconductive symmetry of the model in the sequel. Let's denote the generators of $U_{q}(s u(2))$ with $X^{ \pm}, H$. They satisfy the commutation relations (see e.g. [11]):

$$
\left[H, X^{( \pm)}\right]= \pm 2 X^{( \pm)}, \quad\left[X^{+}, X^{-}\right]=\frac{q^{H}-q^{-H}}{q-q^{-1}}
$$

3.1. Local commutation relations

Definition. Local representation of the superconductive $U_{q}(s u(2))[4]$ at each site $l$ :

$$
\begin{aligned}
& \rho_{s}\left(X^{+}\right) \equiv K_{l}^{(+)}=e^{-i \vec{\Phi} \cdot \vec{p}_{l}} a_{l \uparrow}^{\dagger} a_{l \downarrow}^{\dagger} \\
& \rho_{s}\left(X^{-}\right) \equiv K_{l}^{(-)}=e^{i \vec{\Phi} \vec{p} l_{l}} a_{l \downarrow} a_{l \uparrow}=\left(K_{l}^{(+)}\right)^{\dagger} \\
& \rho_{s}(H) \equiv 2 K_{l}^{(z)}=n_{l \uparrow}+n_{l \downarrow}-1
\end{aligned}
$$

The parameter $\vec{\Phi}$ appearing in (13),(14) does not affect the $U_{q}(s u(2))$-commutation relations, and for the moment it should be regarded as a free variable, which will be determined by the commutation relations with the Hamiltonian. $\frac{\vec{\Phi}}{2}$ can be interpreted as the parameter of a Lang-Firsov transformation [9] of the fermionic operators.

TheOREM 1. The local part of the Hamiltonian commutes with the local generators

$$
\left[K_{l}^{(+)}, H^{(l o c)}\right]=\left[K_{l}^{(-)}, H^{(l o c)}\right]=\left[K_{l}^{(z)}, H^{(l o c)}\right]=0
$$

if and only if the following conditions are satisfied:

$$
\begin{aligned}
\vec{\Phi} & =\frac{2 \vec{\lambda}}{\hbar M \omega^{2}}, \\
\mu & =\frac{u}{2}-\frac{1}{4} M \omega^{2} \hbar^{2} \Phi^{2}=\frac{u}{2}-\frac{\vec{\lambda}^{2}}{M \omega^{2}} .
\end{aligned}
$$

3.2. Global commutation relations. Switching signs on $\rho_{s}\left(X^{ \pm}\right)$gives again a representation of $U_{q}(s u(2))$. It is necessary to consider both representations, i.e.

$$
\rho_{s}^{ \pm}\left(X^{+}\right)= \pm \rho_{s}\left(X^{+}\right), \quad \rho_{s}^{ \pm}\left(X^{-}\right)= \pm \rho_{s}\left(X^{-}\right), \quad \rho_{s}^{ \pm}(H)=\rho_{s}(H),
$$

if the convention is chosen that fermionic operators on different sites anticommute. For each lattice site $l$ a sign $\sigma(l) \in\{1,-1\}$ and the associated representation $\rho_{s}^{\sigma(l)}$ will be 
determined by the symmetry. The local commutation relations are not affected by this choice.

Further, it is necessary to fix some ordering of the lattice sites to be able to define a tensor product and hence to construct a global symmetry.

Definition. Global representation of the superconductive $U_{q}(s u(2))$ [4]:

$$
\begin{aligned}
& K^{(+)}=\bigotimes_{l} \rho_{s}^{\sigma(l)}\left(\Delta^{(N-1)}\left(X^{+}\right)\right)=\sum_{l} \sigma(l) \prod_{r<l} e^{\alpha K_{r}^{(z)}} K_{l}^{(+)} \prod_{r>l} e^{-\alpha^{*} K_{r}^{(z)}}, \\
& K^{(-)}=\bigotimes_{l} \rho_{s}^{\sigma(l)}\left(\Delta^{(N-1)}\left(X^{-}\right)\right)=\sum_{l} \sigma(l) \prod_{r<l} e^{\alpha^{*} K_{r}^{(z)}} K_{l}^{(-)} \prod_{r>l} e^{-\alpha K_{r}^{(z)}}, \\
& K^{(z)}=\bigotimes_{l} \rho_{s}^{\sigma(l)}\left(\Delta^{(N-1)}(H)\right)=\sum_{l} K_{l}^{(z)},
\end{aligned}
$$

$\Delta$ is the coproduct of $U_{q}(s u(2))$ (see e.g. [11]), $\alpha=\ln (q)$ is the deformation parameter, $N$ is the number of lattice sites.

THEOREM 2. The non-local part of the Hamiltonian commutes with the non-local generators

$$
\left[K^{(+)}, H^{(n o n-l o c)}\right]=\left[K^{(-)}, H^{(n o n-l o c)}\right]=\left[K^{(z)}, H^{(n o n-l o c)}\right]=0
$$

if and only if the following conditions are satisfied:

$$
\begin{aligned}
& \sigma(i)=-\sigma(j) \text { for } i, j \text { nearest neighbours, } \\
& 2 \vec{\kappa}=\vec{\Phi} \\
& \operatorname{Re} \alpha=-\vec{R}_{i j} \cdot \vec{\Phi} \zeta \hbar \\
& \prod_{i<r<j} e^{\alpha K_{r}^{(z)}}=\prod_{i<r<j} e^{-\alpha^{*} K_{r}^{(z)}}
\end{aligned}
$$

Remarks to Theorem 2. Eq. (24) imposes that nearest neighbours must have opposite signs. This gives a restriction on the possible lattices, e.g. a triangular lattice could not be chosen.

Eq. (25) fixes the parameter $\vec{\Phi}$ of (13),(14) and relates it to the parameter $\vec{\kappa}$ appearing in the Hamiltonian $H_{H u b}(6)$. As $\vec{\kappa}$ can be interpreted as the parameter of a LangFirsov transformation on the Hamiltonian, while $\frac{\vec{\phi}}{2}$ can be interpreted as the parameter of a Lang-Firsov transformation on the generators of the symmetry, eq. (25) can be interpreted as a consistency relation, requiring the same transformation to be done on the Hamiltonian and on the generators.

Eq. (26) determines the real part of the deformation parameter $\alpha$ of the quantum group. In order for such equation to make sense it is necessary to choose a lexicographic ordering of the lattice sites, so that the sign of the term $\vec{R}_{i j}$ does not depend on the particular couple $i, j$. Notice that there is no restriction on the imaginary part of the deformation parameter, so that we can safely choose it to be real.

Eq. (27) is a strong condition in the case $\alpha \neq 0$. It requires that if $i, j$ is a couple of nearest neighbours then $i<r$ implies $j \leq r$, while $i>r$ implies $j \geq r$ for all $r$. However, 
such a condition implies that the lattice $\Lambda$ on which the Hubbard model is defined is one-dimensional, and that the 'normal' ordering of the sites is chosen, in which the sites are numbered from left to right in increasing or decreasing order.

Proof. (Sketch) The fact that $K^{(z)}$ commutes with $H^{(n o n-l o c)}$ given by (5) is immediate.

We have to calculate

$$
\begin{aligned}
& {\left[K^{(+)}, H^{(\text {non-loc })}\right]=} \\
& t \sum_{\langle i<j\rangle} e^{-\hbar \zeta \vec{R}_{i j} \cdot \vec{\kappa}}\left\{\left(a_{i \downarrow}^{\dagger} a_{i \uparrow}^{\dagger} a_{j \downarrow}^{\dagger} a_{j \uparrow}^{\dagger} a_{i \uparrow} a_{j \downarrow}-a_{i \downarrow}^{\dagger} a_{i \uparrow}^{\dagger} a_{j \downarrow}^{\dagger} a_{j \uparrow}^{\dagger} a_{i \downarrow} a_{j \uparrow}\right)\right. \\
& \times\left[Z_{i j}\left(2 \cosh \left(\frac{1}{2} \vec{R}_{i j} \cdot \vec{\Phi} \zeta \hbar\right)-2 \cosh \left(\frac{1}{2} \vec{R}_{i j} \cdot \vec{\Phi} \zeta \hbar+\frac{1}{2} \alpha^{*}\right)\right)\right. \\
& \left.+Z_{j i}\left(2 \cosh \left(\frac{1}{2} \vec{R}_{i j} \cdot \vec{\Phi} \zeta \hbar\right)-2 \cosh \left(\frac{1}{2} \vec{R}_{i j} \cdot \vec{\Phi} \zeta \hbar+\frac{1}{2} \alpha\right)\right)\right] \\
& +\left(a_{i \uparrow}^{\dagger} a_{j \downarrow}^{\dagger} a_{j \uparrow}^{\dagger} a_{j \uparrow}+a_{i \downarrow}^{\dagger} a_{j \downarrow}^{\dagger} a_{j \uparrow}^{\dagger} a_{j \downarrow}\right) e^{\frac{1}{2} \vec{R}_{i j} \cdot \vec{\Phi} \zeta \hbar}\left[Z_{i j}\left(e^{\frac{1}{2} \alpha^{*}}-1\right)+Z_{j i}\left(e^{-\vec{R}_{i j} \cdot \vec{\Phi} \zeta \hbar} e^{-\frac{1}{2} \alpha}-1\right)\right] \\
& +\left(a_{i \downarrow}^{\dagger} a_{i \uparrow}^{\dagger} a_{j \downarrow}^{\dagger} a_{i \downarrow}+a_{i \downarrow}^{\dagger} a_{i \uparrow}^{\dagger} a_{j \uparrow}^{\dagger} a_{i \uparrow}\right) e^{-\frac{1}{2} \vec{R}_{i j} \cdot \vec{\Phi} \zeta \hbar}\left[Z_{i j}\left(e^{\vec{R}_{i j} \cdot \vec{\Phi} \zeta \hbar} e^{\frac{1}{2} \alpha^{*}}-1\right)+Z_{j i}\left(e^{-\frac{1}{2} \alpha}-1\right)\right] \\
& \left.\left.+\left(a_{i \downarrow}^{\dagger} a_{j \uparrow}^{\dagger}-a_{i \uparrow}^{\dagger} a_{j \downarrow}^{\dagger}\right)\left[Z_{i j} e^{\frac{1}{2} \vec{R}_{i j} \cdot \vec{\Phi} \zeta \hbar} e^{\frac{1}{2} \alpha^{*}}+Z_{j i} e^{-\frac{1}{2} \vec{R}_{i j} \cdot \vec{\Phi} \zeta \hbar} e^{-\frac{1}{2} \alpha}\right)\right]\right\} \\
& +\sum_{l} \sum_{\langle i, j\rangle, i<l<j} \sigma(l) e^{-\hbar \zeta \vec{R}_{i j} \cdot \vec{k}} e^{-i \vec{\Phi} \cdot \vec{p}_{l}} a_{i \uparrow}^{\dagger} a_{l \downarrow}^{\dagger} \prod_{r<l, r \neq i} e^{\alpha K_{r}^{(z)}} \prod_{r>l, r \neq j} e^{-\alpha^{*} K_{r}^{(z)}} \\
& \times\left[e^{\alpha K_{i}^{(z)}} e^{-\alpha^{*} K_{j}^{(z)}}, e^{i \vec{\kappa} \cdot\left(\vec{p}_{i}-\vec{p}_{j}\right)+\zeta \vec{R}_{i j} \cdot\left(\vec{x}_{i}-\vec{x}_{j}\right)} a_{j \uparrow}^{\dagger} a_{i \uparrow}+a_{j \downarrow}^{\dagger} a_{i \downarrow}+h . c .\right] .
\end{aligned}
$$

where we have introduced the abbreviation

$$
Z_{i j}=\sigma(i) e^{-i(\vec{\Phi}-\vec{\kappa}) \cdot \vec{p}_{i}-i \vec{\kappa} \cdot \vec{p}_{j}+\zeta \vec{R}_{i j} \cdot\left(\vec{x}_{i}-\vec{x}_{j}\right)} \prod_{r<i, r \neq j} e^{\alpha K_{r}^{(z)}} \prod_{r>i, r \neq j} e^{-\alpha^{*} K_{r}^{(z)}} .
$$

The sums containing different numbers of fermionic operators must all be separately zero, because they are linearly independent. (We have chosen a normal ordering in the fermionic operators.) By studying the term containing $a_{i \downarrow}^{\dagger} a_{j \uparrow}^{\dagger}-a_{i \uparrow}^{\dagger} a_{j \downarrow}^{\dagger}$, the conditions (24)-(27) are obtained. It turns out that the same conditions guarantee that also the other sums vanish.

\subsection{Summary (see the following table)}

Remark. After choosing an appropriate ordering of the lattice sites for model III) in the 1-dimensional case, we set $\kappa=-\vec{R}_{i j} \cdot \vec{\kappa}$. The models Ib),Ic) and IIa) have no symmetry, because the condition $2 \vec{\kappa}=\frac{2 \vec{\lambda}}{\hbar M \omega^{2}}$ is not satisfied. 


\begin{tabular}{|l|l|}
\hline $\begin{array}{l}\text { Classical Symmetry } \\
D=0\end{array}$ & $\begin{array}{l}\text { Quantum Symmetry } \\
\alpha \neq 0, q=\exp (\alpha) \\
D=1\end{array}$ \\
\hline Id), IIb): $S U(2)_{s}$-symmetry; $\mu=\frac{u}{2}$, & $\begin{array}{l}\text { III): } S U_{q}(2)_{s} \text {-symmetry; } \\
\operatorname{Re} \alpha=2 \kappa \zeta \hbar, \\
\mu=u / 2-\hbar^{2} \kappa^{2} M \omega^{2}, \vec{\lambda}=\hbar M \omega^{2} \vec{\kappa}\end{array}$ \\
$\begin{array}{l}\text { Ia): } S U(2)_{s} \text {-symmetry, } \\
\text { if } \mu=u / 2-\hbar^{2} \vec{\kappa}^{2} M \omega^{2}, \vec{\lambda}=\hbar M \omega^{2} \vec{\kappa}\end{array}$ & \\
Ib),Ic),IIa): no symmetry & \\
\hline
\end{tabular}

4. Discussion. The standard Hubbard model Id) (with decoupled Einstein oscillators) and the Hubbard model IIb) without local phonon interaction $(\vec{\lambda}=0)$ have a superconductive $S U(2)_{s}$ symmetry at "half-filling" $\mu=u / 2$. Two other models Ia), III) with local phonon interaction $(\lambda \neq 0)$ have superconducting symmetries $S U(2)_{s}$ and $S U_{q}(2)_{s}$ respectively at $\mu=u / 2-\hbar^{2} \kappa^{2} M \omega^{2}$. It is well known [9] that a LangFirsov transformation can be performed on the model Ia), shifting the parameters and thereby eliminating the local phonon interaction $\vec{\lambda}$. The resulting Hamiltonian has a $S U(2)_{s}$-symmetry at "half-filling" $\mu=u / 2$. It turns out that a Lang-Firsov transformation with the unitary operator (7) can also be used to eliminate the local electronphonon interaction term from the Extended Hubbard Model. The parameters are shifted according to

$$
\vec{\lambda} \rightarrow \vec{\lambda}-M \omega^{2} \hbar \vec{\kappa}, u \rightarrow u-2 \hbar \vec{\lambda} \cdot \vec{\kappa}+M \omega^{2} \hbar^{2} \vec{\kappa}^{2}, \mu \rightarrow \mu+\hbar \vec{\lambda} \cdot \vec{\kappa}-1 / 2 M \omega^{2} \hbar^{2} \vec{\kappa}^{2}
$$

and the hopping term $H_{e l-p h}^{(n o n-l o c)}$ becomes

$$
t \sum_{\langle i<j\rangle, \sigma} e^{\zeta \vec{R}_{i j} \cdot\left(\vec{x}_{i}-\vec{x}_{j}\right)} q^{\frac{1}{2}} a_{j \sigma}^{\dagger} a_{i \sigma}\left(1+\left(q^{-\frac{1}{2}}-1\right) n_{i,-\sigma}\right)\left(1+\left(q^{\frac{1}{2}}-1\right) n_{j,-\sigma}\right)+\text { h.c. }(30)
$$

The resulting Hamiltonian has a superconducting $S U_{q}(2)_{s}$ quantum symmetry at "half filling" $\mu=u / 2$ (!) with $\vec{\lambda}=0$ and only for $D=1$.

It is even possible to eliminate all phonon terms from this Quantum Symmetric Hamiltonian by a mean field approximation $[8,9]$ without breaking the quantum symmetry. More precisely one performs a thermal average over $H_{p h}$-eigenstates and assumes uncorrelated Einstein oscillators (note that $\vec{\lambda}=0$ !). The exponential in the hopping term is then approximated by a temperature-dependent constant.

Acknowledgements. BLC would like to thank the organizers of the Minisemester on Quantum Groups at the Banach Center and the Forschungszentrum Karlsruhe Technik und Umwelt for financial support. 


\section{References}

[1] J. Hubbard, Proc. R. Soc. London A 276 (1963) 238.

[2] C. N. Yang, S. C. Zhang, Mod. Phys. Lett. B 4 (1990) 759.

[3] C. N. Yang, Phys. Rev. Lett. 63 (1989) 2144.

[4] A. Montorsi, M. Rasetti, Phys. Rev. Lett. 72 (1994) 1730.

[5] B. L. Cerchiai, P. Schupp,On Quantum Groups in the Hubbard Model with Phonons, J. Phys. A, in press.

[6] T. Holstein, Ann. Phys. 8 (1959) 325.

[7] A. L. Fetter, J. D. Walecka, Quantum Theory of Many Particle Systems, (McGraw Hill, New York, 1971) p. 396.

[8] S. Robaszkiewicz, R. Micnas, J. Ranninger, Phys. Rev. B36 (1987) 180.

[9] I. G. Lang, Y. A. Firsov, Sov. Phys. JETP 16 (1963) 1301.

[10] S. Barišić, J. Labbé, J. Friedel, Phys. Rev. Lett. 25 (1970) 919.

[11] M. Jimbo, Lett. Math. Phys. 11 (1986) 247. 